\title{
Quantum limits, nonseparable transformations, and nonlinear optics
}

\author{
Alfredo Luis \\ Departamento de Óptica, Facultad de Ciencias Físicas, Universidad Complutense, 28040 Madrid, Spain
}

(Received 11 March 2007; revised manuscript received 1 July 2007; published 5 September 2007)

\begin{abstract}
We examine the relationship between nonseparable transformations and nonlinear optical processes, surpassing the Heisenberg limit in precision measurements.
\end{abstract}

DOI: 10.1103/PhysRevA.76.035801

\section{INTRODUCTION}

Quantum metrology deals with the fundamental measuring capabilities allowed by the quantum theory. For all measuring schemes the physical variable $x$ to be measured (such as a length, time, force, temperature, etc.) is encoded in a transformation $U_{x}$ experienced by a series of probes. Measurements performed in the final states of the probes allow to infer the value of $x$.

In this context the so-called Heisenberg limit plays a relevant position. It states that the uncertainty $\Delta x$ of the inferred value of $x$ scales as the inverse of the number of particles experiencing the same transformation. Using different approaches it has been argued that this is the ultimate bound to the precision of quantum measurements $[1,2]$. Then, most works in quantum metrology address the probe states, the final measurements, and the proper data analysis required to reach the Heisenberg limit [3].

Furthermore, it is also legitimate to compare the performance of different transformations $U_{x}$ that can be used for the measurement of one and the same physical variable $x$. In some recent works we have shown that the Heisenberg limit applies exclusively to linear transformations and can be surpassed by nonlinear ones [4]. More recent works have shown that entangling transformations may surpass also the Heisenberg limit $[5,6]$.

In this work we compare both approaches to precision measurements surpassing the Heisenberg limit. We show that nonlinear optical transformations provide natural practical realizations of abstract entangling transformations. Moreover, the optical realizations reveal that, under equivalent conditions, nonlinear separable transformations may achieve better precision than entangling ones.

\section{NONSEPARABLE AND NONLINEAR TRANSFORMATIONS}

For the sake of completeness in this section we recall the demonstration that nonseparable transformations allow to beat the Heisenberg limit $[5,6]$. We will consider transformations $U_{x}=\exp [-i \chi(x) H]$, where $\chi(x)$ is a function of $x$, which we will refer to as susceptibility, that depends on the physical processes producing the transformation. The idea is to consider transformations $U_{x}$ that do not factorize as the product of individual probe transformations $U_{x} \neq \prod_{j=1}^{N} U_{x}^{(j)}$, where $U_{x}^{(j)}$ acts exclusively on the probe $j$ and $N$ is the number of probes.

From very general arguments, the uncertainty of $x$, estimated via a linear propagation of uncertainties for simplicity (other strategies would lead to similar conclusions [3,4]), is given by $[1,2,7,8]$

$$
\Delta x=\frac{1}{2\left|\frac{\partial \chi}{\partial x}\right| \sqrt{\nu} \Delta H},
$$

where $(\Delta H)^{2}=\left\langle H^{2}\right\rangle-\langle H\rangle^{2}$ is the variance of $H$ on the collective state of the $N$ probes and $\nu$ is the number of repetitions of the measurement.

The capabilities of nonseparable transformations is demonstrated by the case where the generator $H$ is the product of identical generators $h_{j}$ for the individual probes,

$$
H_{n s}=\prod_{j=1}^{N} h_{j},
$$

which can be compared with the more standard case of separable transformations generated by $H_{\ell}=\Sigma_{j} h_{j}$. Throughout we refer to the transformation generated by $H_{\ell}$ as linear. On the one hand, $H_{\ell}$ is a linear combination of $h_{j}$. On the other hand, $h_{j}$ are usually number operators so that the transformation generated by $H_{\ell}$ produces a linear relation between input and output basic variables, such as position and momentum in mechanics or quadratures in optics. Analogously, $H_{n s}$ is a nonlinear function of $h_{j}$ and generates nonlinear transformations.

Optimum $\Delta x$ implies maximum $\Delta H_{\ell}$ and $\Delta H_{n s}$, which are

$$
\Delta H_{\ell}=\frac{N}{2}\left(\lambda_{M}-\lambda_{m}\right), \quad \Delta H_{n s}=\frac{1}{2}\left(\lambda_{M}^{N}-\lambda_{m}^{N}\right)
$$

where $\lambda_{M}$ and $\lambda_{m}$ are the maximum and minimum eigenvalues of $h_{j}$, respectively, which are reached in both cases by states of the form

$$
|\Psi\rangle=\frac{1}{\sqrt{2}}\left(\prod_{j=1}^{N}\left|\lambda_{M}\right\rangle_{j}+\prod_{j=1}^{N}\left|\lambda_{m}\right\rangle_{j}\right),
$$

which are the analog of the quantum superposition of states (Schrödinger cat states) or maximally entangled (NOON) states [9].

For definiteness let us consider $\lambda_{m}=0$, denoting $\lambda_{M}$ simply by $\lambda$, so that 


$$
\Delta x_{\ell}=\frac{1}{\sqrt{\nu N \lambda}\left|\frac{\partial \chi_{1}}{\partial x}\right|}, \quad \Delta x_{n s}=\frac{1}{\sqrt{\nu \lambda^{N}}\left|\frac{\partial \chi_{N}}{\partial x}\right|},
$$

where we have taken into account that the susceptibilities are in general different for different generators (i.e., for different $N)$ since the underlying physical processes will be different.

The exponential versus linear behavior as functions of $N$ in Eqs. (5) suggests that the uncertainty $\Delta x_{n s}$ may be lesser than $\Delta x_{\ell}$. However, conclusions based on the dependence on $N$ are not valid because of the unknown dependence on $N$ of $\chi_{N}$.

Nevertheless, this lack of knowledge is avoided by focusing on the dependence of the uncertainty in the total number of particles $n$ employed in the measurement for a fixed number of probes $N$. Denoting by $k$ the number of particles in each probe, the total number of particles is $n=N k$. Moreover, it usually holds that $\lambda \propto k=n / N$, since $h_{j}$ are very often number operators. Thus the scalings of $\Delta H$ and $\Delta x$ with the total number of particles $n$ for fixed $N$ are

$$
\Delta H_{\ell} \propto n, \quad \Delta H_{n s} \propto n^{N}
$$

and

$$
\Delta x_{\ell} \propto n^{-1}, \quad \Delta x_{n s} \propto n^{-N} .
$$

We stress that we are comparing the performances of the measurement of the same variable $x$ carried out with two different measuring schemes with fixed $N$ by examining the dependence on the number of particles $n$ conveyed by the input state.

We can appreciate that the accuracy provided by linear transformations is bounded by the Heisenberg limit, while nonseparable ones surpass it. The scaling of $\Delta x_{n s}$ is essentially the same found in Ref. [6]. Moreover, it is worth noting that nonlinear susceptibilities need not be small as demonstrated by the giant nonlinearities arising in the context of coherent multilevel optics [10].

Finally, we point out that the Heisenberg limit can be beaten even by factorized preparations of the probes $\rho$ $=\Pi_{j} \rho_{j}$. If all the probes are prepared in the state with maximum $\Delta h$-i.e., $\Delta h=\langle h\rangle=\lambda / 2$-we get

$$
\Delta H_{n s}=\left(\frac{\lambda}{\sqrt{2}}\right)^{N} \sqrt{1-2^{-N / 2}} \simeq\left(\frac{\lambda}{\sqrt{2}}\right)^{N},
$$

where the last approximation holds for $N \gg 1$.

\section{NONLINEAR OPTICS}

In principle, product Hamiltonians of the form (2) might be regarded as somewhat exotic. However, they do actually arise in the realm of nonlinear optics, so that the two schemes [4] and [5,6] surpassing the Heisenberg limit can be compared on the same grounds.

More explicitly, nonseparable product Hamiltonians (2) arise in light propagation through nonlinear media in the form (for simplicity we embody the $x$ derivative of the susceptibilities $\chi^{\prime}$ with the generators)

$$
H_{n s}=\chi_{n s}^{\prime} \prod_{j=1}^{N} a_{j}^{\dagger} a_{j},
$$

where $a_{j}$ is the complex-amplitude operator of mode $j$. This can be compared with the nonlinear single-mode Hamiltonian (and thus trivially separable or nonentangling) [4]

$$
H_{n \ell}=\chi_{n \ell}^{\prime}\left(a^{\dagger} a\right)^{N} \text {. }
$$

The simplest case $N=2$ of the Hamiltonians (9) and (10) are the cross-Kerr (or cross-phase modulation) and Kerr effects, respectively, which are well known in quantum optics [11].

If we consider the same mean number of photons for the two arrangements, $n=\sum_{j=1}^{N}\left\langle a_{j}^{\dagger} a_{j}\right\rangle=\left\langle a^{\dagger} a\right\rangle$, the maxima of $\Delta H_{n s}$ and $\Delta H_{n \ell}$ are

$$
\Delta H_{n s}=\frac{\chi_{n s}^{\prime}}{2}\left(\frac{2 n}{N}\right)^{N}, \quad \Delta H_{n \ell}=\frac{\chi_{n \ell}^{\prime}}{2}(2 n)^{N},
$$

which both surpass the Heisenberg limit in agreement with Eqs. (6) and (7). The maximum precision is reached by the analogs of the states (4) in the photon-number basis,

$$
\begin{gathered}
|\Psi\rangle_{n s}=\frac{1}{\sqrt{2}}\left(\prod_{j=1}^{N}|0\rangle_{j}+\prod_{j=1}^{N}|2 n / N\rangle_{j}\right), \\
|\Psi\rangle_{n \ell}=\frac{1}{\sqrt{2}}(|0\rangle+|2 n\rangle) .
\end{gathered}
$$

From Eq. (11) we can appreciate that, for the same set of parameters $n$ and $N$, the nonlinear separable transformation provides better resolution than the nonlinear nonseparable.

This is also true in the more practical case of considering factorized semiclassical quadrature coherent states as the states experiencing the transformation $|\Psi\rangle=\Pi_{j=1}^{N}|\beta\rangle_{j}$ and $|\Psi\rangle=|\alpha\rangle$ with $n=N|\beta|^{2}=|\alpha|^{2}$, where $|\beta\rangle$ and $|\alpha\rangle$ are coherent states. In such a case we get

$$
\Delta H_{n s}=\sqrt{N} \chi_{n s}^{\prime}\left(\frac{n}{N}\right)^{N-1 / 2}, \quad \Delta H_{n \ell}=\chi_{n \ell}^{\prime} n^{N-1 / 2} .
$$

Both schemes surpass the Heisenberg limit and here again the nonlinear separable scheme presents a more favorable behavior.

\section{CONCLUSIONS}

Both nonseparable and nonlinear transformations allow us to perform more accurate measurements than separable linear transformations. A suitable arena to implement both of them is nonlinear optics. In this context we have shown that, under equivalent conditions, nonlinear separable transformations present a better performance than nonlinear entangling ones. This result suggests that the key point for surpassing the Heisenberg limit is nonlinearity rather than nonseparability.

These conclusions are significant since they imply a volte- 
face in quantum metrology. This is because they show that it is advantageous to use nonlinear processes to encode signals, instead of using them to generate fragile nonclassical entangled states as input states for linear detection schemes. This idea is accessible in practice via nonlinear optical processes.

\section{ACKNOWLEDGMENTS}

This work has been supported by Project No. PR1-A/0715378 of the Universidad Complutense. I thank the anonymous referees for comments that have helped me to improve the paper.
[1] V. Giovannetti, S. Lloyd, and L. Maccone, Phys. Rev. Lett. 96, 010401 (2006).

[2] W. M. Itano et al., Phys. Rev. A 47, 3554 (1993); D. J. Wineland, J. J. Bollinger, W. M. Itano, and D. J. Heinzen, ibid. 50, 67 (1994); Z. Y. Ou, ibid. 55, 2598 (1997).

[3] J. Combes and H. M. Wiseman, J. Opt. B: Quantum Semiclassical Opt. 7, 14 (2005); Z. Hradil Z and J. Řeháček, Phys. Lett. A 334, 267 (2005).

[4] A. Luis, Phys. Rev. A 65, 025802 (2002); Phys. Lett. A 329, 8 (2004); Phys. Rev. A 69, 044101 (2004); J. Opt. B: Quantum Semiclassical Opt. 6, 1 (2004); J. Beltrán and A. Luis, Phys. Rev. A 72, 045801 (2005).

[5] S. M. Roy and S. L. Braunstein, e-print arXiv:quant-ph/ 0607152.

[6] S. Boixo, S. T. Flammia, C. M. Caves, and J. M. Geremia, Phys. Rev. Lett. 98, 090401 (2007).

[7] S. L. Braunstein and C. M. Caves, Phys. Rev. Lett. 72, 3439 (1994); S. L. Braunstein, C. M. Caves, and G. J. Milburn, Ann. Phys. (N.Y.) 247, 135 (1996).
[8] M. Hillery and L. Mlodinow, Phys. Rev. A 48, 1548 (1993).

[9] N. D. Mermin, Phys. Rev. Lett. 65, 1838 (1990); J. J. Bollinger, W. M. Itano, D. J. Wineland, and D. J. Heinzen, Phys. Rev. A 54, R4649 (1996); A. Luis, ibid. 64, 054102 (2001); 65, 034102 (2002); Ph. Walther, J.-W. Pan, M. Aspelmeyer, R. Ursin, S. Gasparoni, and A. Zeilinger, Nature (London) 429, 158 (2004); M. W. Mitchell, J. S. Lundeen, and A. M. Steinberg, ibid. 429, 161 (2004).

[10] H. Schmidt and A. Imamoğlu, Opt. Lett. 21, 1936 (1996); S. E. Harris and L. V. Hau, Phys. Rev. Lett. 82, 4611 (1999); H. Kang and Y. Zhu, ibid. 91, 093601 (2003).

[11] P. Lambropoulos and D. Petrosyan, Fundamentals of Quantum Optics and Quantum Information (Springer, Berlin, 2007); C. C. Gerry and P. L. Knight, Introductory Quantum Optics (Cambridge University Press, Cambridge, England, 2005); M. O. Scully and M. S. Zubairy, Quantum Optics (Cambridge University Press, Cambridge, England, 1997); L. Mandel and E. Wolf, Optical Coherence and Quantum Optics (Cambridge University Press, Cambridge, England 1995). 